\title{
Vitamin D and coronavirus disease 2019 (COVID-19)—rapid evidence review
}

\author{
William B. Grant ${ }^{1}$ (D)
}

Received: 17 June 2021 / Accepted: 15 July 2021 / Published online: 2 August 2021

(c) The Author(s), under exclusive licence to Springer Nature Switzerland AG 2021

\section{To the Editor:}

The recent review by Raisi-Estabragh and colleagues [1] concluded that evidence to support vitamin D supplementation for the prevention or treatment of COVID-19 is inconclusive. The main reasons given for reaching this conclusion were confounding considerations and reverse causation in observational studies, and limited data from intervention studies. The concerns regarding observational studies are legitimate ones; however, they have already been addressed and found not to affect the findings from observational studies. While there are limited data from intervention studies, vitamin $\mathrm{D}$ intervention studies are difficult to conduct properly and they are not the only way to determine causality. This letter provides important information omitted from this recent review.

Regarding confounding, the review selected a few observational studies to discuss. The proper way to consider confounding is to do a meta-analysis of all available observational studies with adjustment for confounding factors. A recent meta-analysis of three studies found the adjusted odds ratio (aOR) for SARS-CoV-2 infection with vitamin D deficiency $(\mathrm{VDD})=1.77(95 \%$ confidence interval $[\mathrm{CI}]$ $1.24,2.53$ ), which was similar to the result for five studies that did not adjust for confounders ( $\mathrm{OR}=1.75,95 \%$ CI 1.44 , 2.13) [2]. It also found in a meta-analysis of seven studies that VDD was associated with composite severity with an $\mathrm{aOR}=2.57(95 \%$ CI $1.65,4.01)$ vs. $10.6(95 \%$ CI $2.1,54.2)$ for five studies that were not adjusted. Also, as shown in Fig. 4, VDD was associated with mortality for five studies in the Cox survival method (hazard ratio $=7.7,95 \%$ CI 3.9, 15.0), while no association was found for four studies that used logistic regression $(\mathrm{aOR}=1.05,95 \%$ CI $0.63,1.75)$.

William B. Grant

wbgrant@infionline.net

1 Sunlight, Nutrition, and Health Research Center, P.O. Box 641603, San Francisco, CA 94164-1603, USA
For mortality, the combined aOR $=2.50$ (95\% CI 1.34, 4.64). This value was similar for than for five studies that did not adjust for confounders, crude OR 2.62 (95\% CI 1.13, 6.05). Thus, including confounding factors did not greatly affect the ORs for infection, severity, or mortality rates.

Regarding reverse causality, that statement was based on the finding that 25-hydroxyvitamin $\mathrm{D}[25(\mathrm{OH}) \mathrm{D}]$ concentration is lowered by acute inflammatory disease. However, as shown in a recent study involving nine healthy non-smoking male volunteers, the effect lasts only a few hours [3], which is much shorter than the time between COVID-19 infection and hospital admission and blood draw. Further support for reverse causality not being an issue is that aOR for VDD and COVID-19 cases is very similar for studies where $25(\mathrm{OH})$ $\mathrm{D}$ concentration is determined from blood drawn at time of hospital admission or from the preceding year, adjusted for season (see, e.g., https://c19vitamind.com/).

The authors recommended waiting for the results of adequately powered clinical trial data before changing the recommendations regarding vitamin D and COVID-19. There is, however, a more comprehensive approach to determining causality, one outlined by Dr. A. Bradford Hill in his presidential address to the Royal Medical Society in 1965 as discussed in [4] and cited by 9743 publications according to scholar.google.com. The criteria important for vitamin D include strength of association, consistency, temporality, biological gradient, plausibility (e.g., mechanisms), coherence with known facts of biology and the disease, experiment (e.g., randomized controlled trial), and analogy. Accounting for confounding factors was added subsequently. Not all criteria need be satisfied to claim causality, but the more that are, the better. Hill's criteria for causality for vitamin D in reducing risk of COVID-19 were reviewed in Mercola et al. [4] and found to be generally satisfied. The two primary mechanisms are reduction in SARS-CoV-2 virus survival and reduced risk of the cytokine storm through suppression of pro-inflammatory cytokine production. Regarding analogy, it is noted that most vitamin D clinical trials 
were poorly designed and conducted since they were based on vitamin $\mathrm{D}$ dose rather than serum $25(\mathrm{OH}) \mathrm{D}$ concentration [5], often resulting in enrolling participants with high baseline $25(\mathrm{OH}) \mathrm{D}$ concentrations given low vitamin $\mathrm{D}$ doses. The hormonal metabolite of vitamin D, 1,25-dihydroxyvitamin D, produces most of the effects of vitamin D through activating vitamin $\mathrm{D}$ receptors coupled to chromosomes in nearly every cell in the body, thereby regulating expression of many genes. Thus, it is not surprising that a careful search of https://pubmed.ncbi.nlm.nih.gov/ would find a number of vitamin $\mathrm{D}$ supplementation observational studies and clinical trials that demonstrated significant reductions in risk of cancer, diabetes mellitus type 2 , influenza, preterm delivery, and severity of COVID-19 outcomes and that the optimal $25(\mathrm{OH}) \mathrm{D}$ concentration range is $75-150 \mathrm{nmol} / \mathrm{L}$. It should also be noted that evidence-based medicine requires that the best available evidence be used for making decisions. At present, much of the best evidence regarding vitamin D comes from observational studies.

Supplementary Information The online version contains supplementary material available at https://doi.org/10.1007/s40520-021-01941-9.

Funding No funding was received for the preparation of this letter.

\section{Declarations}

Conflict of interest My organization, Sunlight, Nutrition and Health Research Center, receives a research grant for research and education regarding vitamin D from Bio-Tech Pharmacal, Inc. (Fayetteville, AR, USA).

Statement of human and animal rights Not applicable since no human or animal subjects were involved in the preparation of this latter.

Informed consent Not applicable since no humans were involved in any activities regarding the preparation of this letter.

\section{References}

1. Raisi-Estabragh Z, Martineau AR, Curtis EM et al (2021) Vitamin D and coronavirus disease 2019 (COVID-19): rapid evidence review. Aging Clin Exp Res 33:2031-2041

2. Kazemi A, Mohammadi V, Aghababaee SK et al (2021) Association of vitamin D status with SARS-CoV-2 infection or COVID19 severity: a systematic review and meta-analysis. Adv Nutr. https://doi.org/10.1093/advances/nmab012

3. Smolders J, van den Ouweland J, Geven C et al (2021) Letter to the Editor: vitamin D deficiency in COVID-19: Mixing up cause and consequence. Metabolism 115:154434

4. Mercola J, Grant WB, Wagner CL (2020) Evidence regarding vitamin D and risk of COVID-19 and its severity. Nutrients 12(11):3361

5. Grant WB, Boucher BJ, Bhattoa HP, Lahore H (2018) Why vitamin D clinical trials should be based on 25-hydroxyvitamin D concentrations. J Steroid Biochem Mol Biol 177:266-269

Publisher's Note Springer Nature remains neutral with regard to jurisdictional claims in published maps and institutional affiliations. 